\title{
Home Health Care: Reliability and Validity Testing of a Patient-Classification Instrument
}

\author{
Mary Nies Albrecht, Ph.D., R.N.
}

\begin{abstract}
The purposes of this methodologic, descriptive, correlation study were to assess the reliability and validity of the Easley-Storfjell (ES) patient-classification instrument for home care, and determine to what extent it was useful in a large, hospital-based home health care setting. A model for home health care developed by the investigator was used. Interrater reliability and concurrent validity were established by four nurse-raters using a random sample. Nonparametric bivariate correlational analysis demonstrated that the ES instrument was both reliable and valid at a significant level. In addition, there was agreement between the overall ES category rating and rater frequency of home visits. Intrarater reliability for each of the four nurse-raters on a random sample was at a significant level. The results of this study have important implications for home health care agencies providing care to chronically ill patients.
\end{abstract}

Mary Nies Albrecht is Postdoctoral Fellow, Community Health Nursing, University of Michigan, Ann Arbor, Michigan and Associate Professor, Community Health Nursing, Elmhurst College.

This article is based on a part of the dissertation study of the author while at the University of Illinois at Chicago.

This study was supported in part by a grant from Sigma Theta Tau International Honor Society of Nursing. Alpha Lambda Chapter and a grant from the University of Illinois at Chicago, Graduate College.

The author gratefully acknowledges the contributions of the University of Illinois faculty Judith Sullivan, Edwin Chen, Karyn Holm, Robah Kellogg, and Caroline Ross for comments on earlier drafts of this manuscript; the nurses and staff at the Westside Veterans Hospital Based Home Care Department, Chicago. IL for their support of this study; and Dr. Judith Storfjell for her review and critique on the final draft of this manuscript.

Address correspondence to Mary Nies Albrecht, Ph.D., 5504 Groveside Lane, Rolling Meadows, IL 60008.
Home care is preferred by $72 \%$ of the American public over nursing homes for the purpose of promoting, maintaining, or restoring health (De Crosta, 1980). In fact, Shaw (1985) and Myers (1988) predicted that home care and related services will quadruple in volume and number of cases in the next 15 to 20 years. Professional nurses who provide care to patients in their homes must have a reliable and valid method of quantifying the need for care. One such method, a patient-classification instrument, is used to group patients into categories according to the perceived requirements for home care (Giovannetti, 1979).

Several patient classification instruments have been developed for home health care (Churness et al., 1986; Daubert, 1979; Hardy, 1984; Harris, Santoferraro, \& Silva, 1985; Martin, 1982). These instruments base patient classification on areas such as rehabilitation potential, nursing diagnosis, or amount of skilled nursing care required for specific procedures. The Easley-Storfjell (ES) instrument is more comprehensive and includes a broader sample of variables for classification (Allen, Easley, \& Storfjell, 1986): (1) clinical judgment; (2) teaching needs; (3) physical care; (4) psychosocial needs; (5) multiagency involvement; and (6) number and severity of problems (Fig. 1). It is also easy to use and takes a limited amount of time to complete.

A major problem with some of the classification instruments assessed, including the ES instrument, is that their reliability and validity have not been established. Both qualities must be determined before any instrument can be used with confidence. Measuring client nursing care needs is the first step in determining what elements would be helpful for staffing decisions and caseload planning in home care. The purposes of this study were to assess the reliability and validity of the ES patient-classification instrument, as well as the ex- 
tent this instrument was useful in a large institutional hospital-based home care setting (HBHC).

\section{SIGNIFICANCE OF THE STUDY}

In the future, prospective payment will be used in the home health care field, and patient classification will be important for accurate costing and for providing quality care. The diagnoses-related group (DRG) method used by hospitals categorizes patients according to acute care and medical needs. However, the needs of home care patients differ greatly from those of institutionalized patients; generally, home care patients do not need medical care, but they do require nursing care as well as other recuperative assistance such as physical therapy or a home health aide. Therefore, prospective payment should classify these patients according to their actual home care needs and not their medical needs.

As these social and economic forces continue to drive growth in the home care industry, patient classification can be used for administrative decision making regarding staffing, patient care evaluation, and the planning of daily nursing assignments. Grouping patients into valid categories provides a rational approach to allocation of nursing care resources and may eliminate inefficiencies that can slow down referrals to home care. It also can assist both staff and management in the process of utilization review (Engstrom, 1987).

On review of instruments for patient classification in home care, three issues become obvious: first, the instruments must be tested for reliability and validity; second, researchers have to use and test the existing instruments rather than contribute new ones to the literature without determining their reliability and validity (Verran, 1986); and third, prospective payment will be moving to home care and a reliable and valid patientclassification system must be in place (Buck \& Harris, 1987; Mitty, 1987). For these reasons it will be important for nursing to have information on home health care patient needs based on reliable and valid measures.

A model for home health care developed by the investigator was used for this study (Albrecht, 1990). It identifies the relationship among structure, process, and outcome variables in the home health care system. This study looked at two selected variables from the model, the relationship between patient classification and number of home visits.

\section{RESEARCH STUDY QUESTIONS}

The review of literature on home health care patient classification led to the following questions:

1. Is there a relationship between overall category rating when using the ES prototype system and a factor version of the instrument for the classification of the same patient?

2. What is the interrater equivalence reliability of the instrument?

3. What is the intrarater reliability of the instrument?

4. What is the relationship of the physical care variable of the ES instrument to the Katz activities of daily living (ADL) scale?

5. Which of the six ES variables has the highest correlation with the overall category rating?

6. What is the relationship between overall category rating (patient classification) and the frequency of home visits as determined by the rater and the staff nurse?

\section{METHODS}

\section{Design and Setting}

This was a methodologic, descriptive, correlational study in an HBHC program in a Veterans Administration (VA) hospital in the Midwest. There are $172 \mathrm{VA}$ hospitals in the United States with a total of $64 \mathrm{HBHC}$ departments. The types of patients at each HBHC vary according to the HBHC focus and population served. The majority have chronic illness, with the exception of one HBHC that focuses on oncology.

The VA hospital where data collection occurred is a 519-bed general medical-surgical center. The HBHC was established in 1985, with the capacity for a census between 50 and 60 patients at any one time. Patients are accepted on an availability basis. The majority of referrals are from the hospital, although some come from the clinics, outpatient department, and the nursing home. The number of referrals received during the year was 58 and the number accepted into the HBHC program was 38 . Since the majority of patients have chronic disease and require long-term home care, the turnover is low.

The HBHC department has two nurses prepared with master's degrees in nursing who each carry approximately 25 to 30 patients in their caseloads. Both are responsible for making assessment visits on patients in their caseload as well as continuing home visits on a frequency based on their professional judgment.

\section{Population and Sample}

The estimated sample size (statistical estimate) was doubled to allow for greater confidence in the calculated scores, resulting in a random sample of 30 active patient records from the study site. The standardized nursing admission flow sheet and standardized admission charting form on the progress notes was used for analysis. By using patient records, the same documentation could be obtained for each patient. 
The random sample was selected using a table of random numbers (Shelley, 1984), with allowance for sampling with replacement. If one of the patient records did not contain all the necessary information, another could be selected randomly. These 30 active patients represented a random sample of the 60 possible active patients in the HBHC. Active records were used since the predicted frequency of home visits was available only for them. All names and identifying information were deleted from records before use. Each record was assigned a code number.

\section{Establishment of Reliability and Validity}

Four raters were used to establish reliability and validity. The Katz index of ADL was used to test concurrent validity. This measure was chosen because it is one of the best-known and most carefully studied ADL tests (Kane \& Kane, 1981). In addition, it covers all the categories of patients in this HBHC program. Specifically, the Katz measure was used to test concurrent validity of the physical care variable of the ES instrument.

The Katz ADL calls for a rating of six functions: bathing, dressing, going to the toilet, transfer, continence, and feeding. The coefficients of reproducibility were 0.948 and 0.976 (Kane \& Kane, 1981, p. 45). Each item is assigned points according to a defined decision rule ( 1 = uses a device; 2 = needs human assistance; 3 = completely dependent). The sum of all the items is then used to describe ADL activities.

The ES instrument is a prototype measure. It requires raters to compare the actual characteristics of a patient with those that most closely match the prototype or overall category description based on the nurses judgment. To determine case difficulty or acuteness of need, each patient is assessed in terms of difficulty based on the six variables in the ES instrument: clinical judgment, teaching needs, physical care, psychosocial needs, multiagency involvement, and number and severity of problems. These variables have been specified into four groupings of magnitude from minimal (1) to very great (4). The prototype category classification is based on the highest numerical rating (most difficult) in which the patient meets two or more of the six criteria. For example, if a patient received a value of $2,1,3,2$, 2 , and 1 in the six areas, the prototype rating would be 2 , indicating moderate difficulty.

An alternative scoring method (factor method) developed by the investigator uses the mean score by summing the ratings and dividing by the total number of ratings. To provide a further check on the reliability and to guard against observer drift (Shelly, 1984), intrarater reliability was determined at the end of the study. Each of the four raters rerated six charts that were randomly selected from the total sample.

\section{Data Collection Procedures}

According to the writer, a new graduate of a baccalaureate nursing (BSN) program was considered to have the appropriate knowledge base to understand and rank patients according to the ES and the ADL instruments. In fact, all four raters had a BSN and 7 to 16 years of nursing experience $(\bar{X}=11.3)$.

A two-day training session was conducted, consisting of orientation to the data collection procedures, instructions on scoring the two instruments, practice sessions, discussion, feedback, and pilot test. The pilot test $(N=10)$ on closed records validated that necessary information was available in the admission charting to complete the instruments. The pilot-tested patient records were not part of the main study. Each of the four raters completed the ES and the Katz tools using the admission visit for each of the $\mathbf{3 0}$ randomly drawn patient charts. The investigator stressed the importance of treating each chart separately and rating each patient assessment visit independent of all others. The four raters were instructed to use both instruments on the same day to help prevent external variables from affecting their ratings, and to alter the instrument used first by flipping a coin. It was not necessary for all raters to be present on the same days since the data did not change.

\section{RESULTS}

\section{Sociodemographic Features}

The mean age of the sample was 71.4 years (median $=$ $69 \mathrm{yrs}$, mode $=65$, range $=59-95 \mathrm{yrs}$ ). Seventy-seven percent were 65 or older. All of the patients in the sample were men; 7 were white, 21 black, 1 Hispanic, and 1 Asian. Fifty percent were married and $80 \%$ lived with someone. The fact that $80 \%$ of the patients receiving home care had a caregiver living with them, with $67 \%$ of these caregivers having minimal or no limitations, has implications for patient classification. It was not surprising that the hospital was the most frequent agency last providing care (50\%) since most of the $\mathrm{HBHC}$ referrals came from the hospital. Table 1 shows the frequency distribution of nursing and medical diagnoses. Ineffective breathing pattern, activity intolerance, and impaired mobility accounted for $53 \%$ of the nursing diagnoses. This is consistent with Hardy's (1984) findings. Cerebrovascular accidents, arteriosclerotic heart disease, and chronic obstructive pulmonary disease accounted for $67 \%$ of the medical diagnoses, consistent with the findings of Brill, Scholosser, and Widmer (1978). 
TABLE 1. Frequency Distribution of Primary Nursing Diagnosis and Primary Medical Diagnosis $(n=30)$

\begin{tabular}{lcc}
\hline Diagnosis & Frequency & Percentage \\
\hline Nursing diagnosis & & \\
$\quad$ Breathing pattern, ineffective & 6 & 20.0 \\
Activity intolerance & 6 & 20.0 \\
Mobility, impaired physical & 4 & 13.3 \\
$\quad$ Knowledge deficit: diet, & 4 & 13.3 \\
$\quad$ medications, signs and & & \\
$\quad$ symptoms & & \\
$\quad$ Nutrition, alteration in & 3 & 10.0 \\
Self-care deficit: activities of & 3 & 10.0 \\
$\quad$ daily living & & \\
Noncompliance: diet \& & 2 & 6.7 \\
$\quad$ medications & & \\
Cardiac output, alteration in & 2 & 6.7 \\
Medical diagnosis & & \\
Cerebrovascular accident & 8 & 26.7 \\
Arteriosclerotic heart disease & 7 & 23.3 \\
Chronic obstructive pulmonary & 5 & 16.7 \\
$\quad$ disease & & \\
Alzheimer's disease & 3 & 10.0 \\
Diabetes & 3 & 10.0 \\
Cancer & 2 & 6.7 \\
Organic brain syndrome- & 2 & 6.7 \\
$\quad$ dementia & & \\
\hline
\end{tabular}

\section{Category Rating Prototype versus Factor}

Because of ordinal level data, the Spearman rank correlation $\left(r_{s}\right)$ and Kendall's Tau correlation $(T)$ were used to evaluate the strength of the relationship between the prototype and the factor ratings. Both Spearman and Kendall's correlations are provided. Spearman is more frequently reported in the literature, however, Kendall provides a more conservative correlation measure. The Spearman rank correlation revealed a substantial significant relationship between the prototype rating (highest category with at least 2 ratings) and the factor rating (6 ratings added and divided by 6$)\left(r_{s}=0.8670 ; P<\right.$ 0.001 ). The Kendalls' Tau correlation was also significant and strong $\left(\mathrm{t}_{\mathrm{c}}=0.7535 ; P<0.001\right)$.

\section{Interrater Reliability}

To test the overall agreement among the four raters, Kendall's coefficient of concordance (W) was used. This test is suited to determining overall agreement for ordinal-level data. The sample consisted of 30 patient charts rated independently by four nurse raters. Interrater reliability was determined for both the prototype and factor rating methods. The prototype agreement was significant at $P<0.0020$. The strength of the agreement was $W=0.4817$. The factor agreement was significant at $P<0.0001$. The strength of the agreement
TABLE 2. Intrarater Reliability $(n=24)$

\begin{tabular}{lrc}
\hline & \multicolumn{2}{c}{ Correlation Coefficient } \\
\cline { 2 - 3 } Ratings & \multicolumn{1}{c}{ Spearman } & Kendall \\
\hline Factor rating & 0.8287 & $0.6799^{*}$ \\
& $P<0.001$ & $P<0.001$ \\
Prototype rating & 0.6112 & $0.5908^{\dagger}$ \\
& $P=0.0020$ & $P=0.0012$ \\
ADL rating & 0.9520 & $0.8304^{*}$ \\
Time/number of visits & $P<0.001$ & $P<0.001$ \\
& 0.7968 & $0.7490^{\dagger}$ \\
& $P<0.001$ & $P<0.001$ \\
\hline
\end{tabular}

*Kendall's $\mathrm{Tau}_{\mathrm{c}}$.

†Kendall's Tau ${ }_{\mathrm{b}}$.

among the four raters was stronger for the factor than for the prototype method $(W=0.5749)$.

\section{Intrarater Reliability}

Intrarater reliability was calculated after the main study was completed. The investigator supervised the raters individually while they evaluated six patient charts randomly selected from the total sample. This was done on four separate occasions so that each rater had the total sample to select charts. Each of the four raters was instructed to rerate the six charts independently (Table 2 ). Katz ADL rating and the number of visits were also checked. The highest intrarater correlation was noted in the Katz ADL rating. This is not surprising, since ADLs are concrete and defined activities, whereas the factor rating, prototype rating, and time/number of visits included a number of less defined variables.

\section{Relationship of Physical Care to Katz ADL}

This relationship was assessed to determine the concurrent validity of the ES instrument. It was expected that the physical care variable would have a high positive correlation with the physical care variable of the Katz ADL scale, since they both measure patients' ability to perform activities of daily living.

Again, both the Spearman rank correlation test and Kendall's Tau correlation were used to evaluate the strength of the relationship. The strength of the relationship was measured by $(\mathrm{T})$ was moderately strong $\left(\mathrm{t}_{\mathrm{c}}=\right.$ $0.5458 ; P<0.001$ ) and the $r_{s}$ relationship slightly stronger $\left(r_{s}=0.6380 ; P<0.001\right)$. Both findings were significant beyond the 0.001 level, as expected.

\section{Variable Correlation with Overall ES Rating}

All variables were significantly correlated with the overall ES category rating with correlations beyond the 0.001 level of significance. Using the prototype method, 
TABLE 3. Summary of Correlation of Easley-Storfjell Difficulty Variables with Factor Scoring and Prototype Scoring $(n=30)^{*}$

\begin{tabular}{|c|c|c|}
\hline Difficulty Variable & $\begin{array}{l}\text { Factor Score } t \\
\left(r_{s}\right)\left(t_{c}\right)\end{array}$ & $\begin{array}{l}\text { Prototype } \\
\text { Scoring } \neq \\
\left(r_{s}\right)\left(t_{c}\right)\end{array}$ \\
\hline $\begin{array}{l}\text { Number, severity of } \\
\text { problems }\end{array}$ & $(0.7644)(0.7010)$ & $(0.6422)(0.6588)$ \\
\hline Clinical judgment & $(0.7419)(0.6280)$ & $(0.6724)(0.6429)$ \\
\hline Psychosocial needs & $(0.7304)(0.6280)$ & $(0.7054)(0.6588)$ \\
\hline Physical care & $(0.7021)(0.5694)$ & $(0.5364)(0.5119)$ \\
\hline $\begin{array}{l}\text { Multiagency } \\
\text { involvement }\end{array}$ & $(0.6806)(0.6590)$ & $(0.4744)(0.4435)$ \\
\hline Teaching needs & $(0.6274)(0.5207)$ & $(0.6418)(0.6283)$ \\
\hline
\end{tabular}

*All significant beyond the 0.001 level.

†Factor $=$ Kendall's Tau ${ }_{c}$.

$\ddagger$ Prototype $=$ Kendall's Tau $_{b}$.

psychosocial needs $\left(r_{s}\right.$ and $t_{b}$ ) had the highest correlation. Using the factor rating method, number and severity of problems $\left(r_{s}\right)$ and psychosocial needs $\left(t_{b}\right)$ had the highest correlation with the overall ES category rating. A summary of the correlation of the six ES difficulty variables with the factor scoring and prototype scoring method is shown in Table 3.

\section{Category Rating and Frequency of Visits}

\section{Rater}

Frequency of visits was determined by using the time determination of the ES instrument (Fig. 1). The four raters determined the number of visits that they felt were necessary immediately after the difficulty determination for each patient was decided. A significant relationship existed between the overall ES category rating and the frequency of visits (factor $t_{c}=0.5570, P<$ $0.001 ; \mathrm{r}_{\mathrm{s}}=0.6436 ; P<0.001$ ) (prototype $\mathrm{t}_{\mathrm{b}}=0.5450$, $\left.P<0.001 ; r_{s}=0.5800, P<0.001\right)$. As each rater marked the frequency of visits after determining a patient's classification category, the investigator anticipated a positive correlation.

\section{Staff Nurse}

In addition to determining the rater correlation of frequency of visits to overall ES category rating, the investigator looked at the relationship between the overall ES category rating determined by the four raters and the staff nurse's determinations of the frequency of visits on the admission assessment visit. The frequency of visits was determined and documented by the staff nurse on the chart after the initial admission visit. This information was collected by the investigator and was not made available to the raters in their data-collection process. The results showed a negative relation $\left(\mathrm{t}_{\mathrm{c}}=\right.$ $0.1256, P=0.0536 ; \mathrm{r}_{\mathrm{s}}=-0.1519, P=0.098$ for factor and $\mathrm{t}_{\mathrm{b}}=-0.1109, P=0.0969 ; \mathrm{r}_{\mathrm{s}}=-0.1203, P=$ 0.191 for prototype). However, the results were not statistically significant at the 0.05 level, and therefore the findings are questionable.

\section{DISCUSSION}

A highly significant correlation between the factor and prototype methods of rating $(0.75)$ was found. Holm and Llewellyn (1986) stated that an $r$ value of 0.70 to 0.90 can be interpreted as a high correlation. Therefore, it is acceptable to use the ES system as a factor or prototype version for patient classification. The results indicated significant interrater agreement with moderately high interrater strength among the four raters. However, the factor scoring method produced a higher reliability coefficient $(0.60)$ than the prototype method $(0.50)$. It may be that the factor rating is more sensitive because of the use of decimal places. A reliability coefficient of 0.60 to 0.70 is acceptable if one is interested in making group level comparisons (Polit \& Hungler, 1987). When measures are used as a basis for making critical decisions about individuals, however, the reliability coefficient should be 0.90 or better (Polit \& Hungler, 1987).

Several reasons possibly account for the moderately significant correlation. First, although each rater had the same educational background, each had different clinical experiences. Churness et al. (1986) noted in their study of interrater reliability that the nurse-investigator and the nurse-data collector had different perceptions of what was being taught. It may be that clinical judgment varies with type of experience. Second, the difficulty determination of multiagency involvement had low correlation with the overall ES category rating, which may have decreased the interrater reliability. Third, the present ES category descriptors are possibly not specific enough and should be refined further by developing specific cues or indicators as to what they mean. Fourth, the instrument may benefit from inclusion of key words or descriptors in the instrument itself.

The second reliability question was related to intrarater reliability of the instrument. Based on acceptable reliability coefficients of 0.70 or above, three of the four areas were acceptable (factor scoring, ADL, time/number of visits). The prototype scoring was not acceptable. The time interval for checking intrarater reliability ranged from 1 to 17 days. This is an important finding because it says that all four raters were in high agreement with rerating a random sample of charts. Regarding concurrent validity, the ES instrument physical care variable had significant correlation with the ADL scale. In addition, the ES instrument was developed by nurse experts in home care, thus establishing face validity. 


\section{Time Determination}

1. Monthly or less; only one visit

2. Biweekly

3. 1-2 times per week

4. 3-5 times per week
Note: Extensive followup or lengthy visits (over $1 \frac{1}{2}$ hrs) add one time level

Brief visits (under $1 / 2$ hrs) subtract one time level

\section{Difficulty Determination}

Assign the highest numerical categorical rating (most difficult) in which the case meets two or more of the criteria. Based on:
a. Clinical judgment
b. Teaching needs
c. Physical care
d. Psychosocial needs
e. Multiagency involvement
f. number and severity of problems

1. Minimal

a. Requires limited judgment, use of common sense, observation of fairly predictable change in patient status.

b. Requires basic health teaching.

c. Requires no or simple maintenance care.

d. Requires ability to relate to patients and families.

e. Requires limited involvement of only one other agency/provider.

f. Few or uncomplicated problems.

2. Moderate

a. Requires use of basic problem-solving techniques, ability to make limited patient assessments.

b. Requires teaching related to common health problems.

c. Requires basic rehabilitation or use of uncomplicated technical skills. d. Requires use of basic interpersonal relationship skills.

e. Requires limited involvement of two other agencies/providers.

f. Several problems with limited complexity.

3. Great

a. Requires use of well-developed problem-solving skills enhanced by comprehensive knowledge of physical and social sciences, ability to make patient and family assessments.

b. Requires teaching related to illness, complications, and/or comprehensive health supervision.

c. Requires use of complicated technical skills.

d. Requires professional insight and intervention skills in coping with psychosocial needs.

e. Requires extensive involvement of at least one other agency/provider or coordination of several agencies/providers.

f. Several complicated problems.

4. Very great

a. Requires use of creativity, ability to initiate and coordinate plan for patient or family care, use of additional resources and increased supervisory support, ability to make comprehensive patient and family assessment.

b. Requires teaching related to unusual health problems or teaching-learning difficulties.

c. Requires knowledge of scientific rationale that underlies techniques, and ability to modify care in response to patient/family need.

d. Requires ability to intervene in severe psychosocial problems.

e. Requires extensive coordination of several agencies/providers.

f. Numerous or complicated problems requiring augmentation of the knowledge base.

Figure 1. Easley-Storfjell instruments for caseload/workload analysis. (Reproduced with permission of the author, J. Storfjell.)

The Spearman rho (factor and prototype) correlation was in agreement as to the three highest correlations with overall difficulty rating. The top three were number and severity of problems, psychosocial needs, and clinical judgment. Kendall's Tau was not in agreement as to the three highest correlations. The psychosocial needs variable was in the three highest for both factor and prototype ratings. The investigator expected to find significant correlations between the six ES variables and the overall ES category rating. However, it was interesting that psychosocial needs had the highest correlation with the overall ES category rating. This may demonstrate the importance of family and support systems to patients receiving home care. This was an unexpected finding.

Number and severity of problems (factor) and clinical judgment (prototype) were only listed by one rating. An interesting finding was that multiagency involvement and teaching needs were in the three highest for Kendall's Tau ratings and were in the lowest three for Spearman's rho correlation. The results are inconsistent and need further clarification regarding substantive or statistical issues. Factor analysis on the six ES variables for the purpose of reducing a large set of variables into 
a smaller, more manageable set of measures might resolve the question of the need for multiagency involvement as one of the categories.

For the four raters, frequency of visits and overall ES category rating had a significant moderately strong correlation. For the staff nurse frequency of visits and correlation with overall ES category rating, the expected result was not obtained. The values indicated a low negative correlation between the two. The correlation was not significant, however, and therefore the findings are questionable. The low negative correlation does suggest that as the staff nurse frequency of visits goes up the overall ES category rating goes down. Some possible reasons for the negative relationship are that (1) the difficulty and time ratings of the ES instrument are independent and therefore one does not expect one rating to correlate or predict the other rating, (2) the staff nurse was not using the six ES categories in her decision as to number of visits, (3) the staff nurses in the agency have master's degrees, whereas the raters had BSNs, and (4) in this agency there are no limits on the number of visits the staff nurse may make.

In fact, 19 of the patients were seen once a week by the staff nurse, 7 were visited biweekly, and 4 were visited monthly. The judgment by which the staff nurse determines the number of home visits requires further study. Research investigations as to the subjective criteria nurses use in making their decisions may provide explanations for these differences. A useful study would be to have the staff nurse rate the patients using the ES instrument based on the chart admission data and compare the rater and staff nurse findings. Controls for education and experience would also be beneficial.

Other uses of the instrument are to determine costs per category for each of the six variables. Costs could be calculated for overall category rating and then related to various patient variables as well as nursing diagnosis. Since nursing diagnoses reflect only part of the total cost of nursing care (Buck \& Harris, 1987), it is important to include other variables such as those in the ES instrument.

\section{CONCLUSION AND RECOMMENDATIONS}

The results of this study must be considered in view of the limitations. First, the subjects were all male and primarily black. Second, because chronic illness was the major diagnosis, the acuteness of illness of these patients may not represent that of other non-VA populations. Also, there were no limits on the number of home visits the staff nurse made. The generalizability of this study is limited by the fact that all data were collected in one VA hospital.

Recommendations on reconstruction of the instru- ment based on this study are as follows. First, in the time determination, the number of visits per week (3-5) should be examined. Because the majority of home health care patients have chronic illness, they may require more intensive and longer duration of care (Lampley \& Freeman, 1984). This number was selected only three times by three different raters for three different patients. Second, the category "very great" should be examined since it was chosen only twice for clinical judgment, three times for teaching needs, and once for physical care. It was not selected for psychosocial needs, multiagency involvement, or number and severity of problems. Categories that few patients require should be eliminated (Johnson, 1984; Van Slyck, 1985; Verran, 1986).

A final recommendation is to develop indicators of each of the six difficulty variables to enhance understanding of their definition and difference between each level. Replication of this study in settings other than the VA is suggested to validate further a classification system for home health care patients.

\section{REFERENCES}

Albrecht, M.N. (1990). The Albrecht nursing model for home health care: Implications for research, practice, and induction. Public Health Nursing, 7(2), 118-126.

Allen, C., Easley, C. , \& Storfjell, J. (1986). Cost management through caseload/workload analysis. In F. Shaffer (Ed.), Patients and purse strings (pp. 331-346). New York: National League for Nursing.

Brill, R., Scholosser, A., \& Widmer, C. (1978). Home health care services and costs. Nursing Outlook, 26, 488-493.

Buck, J., \& Harris, M. (1987). Costing home health care. Home Healthcare Nurse, 5(6), 17-29.

Churness, V.H., Kleffel, D., Jacobson, J., \& Onodera, M. (1986). Development of a patient classification system for home health nursing. In F.A. Shaffer (Ed.), Patients and purse strings (pp. 319-330). New York: National League for Nursing.

Daubert, E.A. (1979). A patient classification system and outcome criteria. Nursing Outlook, 6, 450-454.

De Crosta, T. (1980). Home health care: its red hot and right now. Nursing Life, (March-April), 2, 54-60.

Engstrom, M. (1987). Home health utilization review. Home Healthcare Nurse, 5(6), 30-33.

Giovannetti, P. (1979). Understanding patient classification systems. Journal of Nursing Administration, 9(2), 4-9.

Hardy, J.A. (1984). A patient classification system for home health patients. Caring, 3(8), 26-27.

Harris, M.D., Santoferraro, C., \& Silva, S. (1985). A patient classification system in home health care. Nursing Economics, 3, 276-282.

Holm, K., \& Llewellyn, J. (1986). Nursing research for nursing practice. Philadelphia: W.B. Saunders.

Johnson, K. (1984). A practice approach to patient classification. Nursing Management, 15(6), 39-46. 
Kane, R.A., \& Kane, R.L. (1981). Assessing the elderly. Lexington, MA: D.C. Heath.

Lampley, P., \& Freeman, R. (1984). Utilization and referral patterns for home health services: $A$ data base for needs determination. Home Health Services Quarterly, 5(1), 89106.

Martin, K. (1982). A client classification system adaptable for computerization. Nursing Outlook, 30, 515-517.

Mitty, E. (1987). Prospective payment and long-term care: Linking payment to resource use. Nursing and Health Care, 8(1), 15-21.

Myers, M. (1988). Home care nursing: A view from the field. Public Health Nursing, 5(2), 65-67.
Polit, D., \& Hungler, B. (1987). Nursing research: Principles and methods (3rd ed.). Philadelphia: J.B. Lippincott.

Shaw, S. (1985). A home care technology. Caring, 3, 21-25.

Shelley, S. (1984). Research methods in nursing and health. Boston: Little, Brown.

Van Slyck, A. (1985). Nursing services: Costing, pricing, and variable billing. In F. Shaffer (Ed.), Costing out nursing: Pricing our product (pp. 39-53). New York: National League for Nursing.

Verran, J. (1986). Patient classification in ambulatory care. Nursing Economics, 4(5), 247-251. 